\title{
Expressions of Putative Cancer Stem Cell Markers, CD44 and CD133, are Correlated with Pathological Tumour Stage in Gastric Adenocarcinoma
}

\author{
Selma Erdogan Duzcu, Hesna Muzeyyen Astarci, Nur Tunc and Cetin Boran \\ Department of Pathology, Abant Izzet Baysal University Medical School, Turkey
}

\begin{abstract}
Objective: To analyse partial and total gastrectomy specimen in items of immunohistochemical staining of the stem cell CD44 and CD133; and to determine their relationship to pathological stage, other clinicopathological prognostic parameters, and their predictive value.

Study Design: Derscriptive study.

Place and Duration of Study: Department of Pathology, Bolu Abant Izzet Baysal University, Medical School, Turkey, from 2013 to 2018.

Methodology: Eighty-three cases, diagnosed with adenocarcinoma from stomach partial and total gastrectomy slides and blocks, were included in the study. Adenocarcinoma cases, that had received neoadjuvant treatment before gastrectomy, cases diagnosed with lymphoma and other malignancies and cases of resection performed for reasons other than tumours, were excluded. Formalin-fixed and paraffin wax-embedded gastric adenocarcinoma blocks were sliced. CD44 and CD133 were stained onto the slides in a Leica Bond Max staining device in compliance with immunohistochemical staining datasheets. Membranous staining for CD44 and cytoplasmic, membranous or luminal staining for CD133 were evaluated. Mann-Whitney U-test and Kruskal- Wallis test were used for group comparisons. For pairwise comparisons, post-hoc Dunn's tests were used. The results were assessed on the significance level of $p<0.05$. The analyses were performed using the Statistical Package for the Social Sciences version 22.0 for Windows (SPSS Inc., Chicago, Illinois, USA).

Results: Considering CD44 and CD133 expressions in terms of pathological stage, there was significantly more intense expression in the T3 and T4 cases ( $p=0.009$, and $p=0.002$, respectively).

Conclusion: Although CD44 is regarded to be associated with more prognostic parameters compared to CD133, both immunohistochemical stains were shown to be related to pathological stage. Thus, these may be guiding for determining the tumour depth.
\end{abstract}

Key Words: Gastric adenocarcinoma, CD44, CD133, Stem cell marker, Prognosis.

How to cite this article: Duzcu SE, Astarci HM, Tunc N, Boran C. Expressions of Putative Cancer Stem Cell Markers, CD44 and CD133, are Correlated with Pathological Tumour Stage in Gastric Adenocarcinoma. J Coll Physicians Surg Pak 2021; 31(05):553-558.

\section{INTRODUCTION}

Gastric adenocarcinoma is the second cause of cancer-related deaths and the fourth most common cancer in the world. ${ }^{1-3}$ According to the 2014 data from the Turkey United Database, gastric adenocarcinoma is the 5 th most common cancer among men $(14.3 \%)$ and the 6 th most common among women $(6.5 \%){ }^{4}$

Correspondence to: Dr. Selma Erdogan Duzcu, Department of Pathology, Abant Izzet Baysal University Medical School, Turkey

E-mail: serdoganduzcu@hotmail.com

Received: January 04, 2021; Revised: March 11, 2021;

Accepted: March 27, 2021

DOI: https://doi.org/10.29271/jcpsp.2021.05.553
Gastric cancer stem cells are transformed from adult tissue-specific stem cells, located in the gastric isthmus/corpus neck glands. ${ }^{5} \mathrm{CD} 44$ and $\mathrm{CD} 133$ are cancer stem cells, isolated from solid tumours. ${ }^{6}$ The CD44 family takes a role in the epithelial mesenchymal transition of proteins in gastric tumours, DNA repair, invasiveness and metastasis. ${ }^{7,8}$ However, the clinical and prognostic role of CD44 variants on gastric cancer is not clear. $^{7}$

CD133 is associated with poor prognosis in gastric cancer and may be used as a therapeutic agent in gastric cancer. ${ }^{5} \mathrm{CD} 133--$ positive tumours are more aggressive in prostate and colon carcinoma, and there are few studies on tumour biology, metastasis and recurrence in gastric cancer. ${ }^{2,9}$

The aim of this study was to determine the relationship between the expressions of the cancer stem cell markers CD44 and 
CD133 in gastric adenocarcinomas and the pathological stage and proven prognostic significant, to emphasise the prognostic and predictive significance and to shed light on determination of targeted treatment approaches.

\section{METHODOLOGY}

This study was conducted with a retrospective design. The partial and total gastrectomy materials of 83 patients, who were admitted to the Department of Pathology, Bolu Abant Izzet Baysal University School of Medicine, between 2013 and 2018, were found in the archive records. The patients did not receive chemotherapy and radiotherapy before the operation. Cases that received a diagnosis of adenocarcinoma from stomach partial and total gastrectomy materials were included in the study. Adenocarcinoma cases that had received neoadjuvant treatment before gastrectomy, cases diagnosed with lymphoma or other malignancies, and cases of resection performed for non tumour reasons, were excluded. The clinical findings of the patients were obtained from the archive records. Histopathological parameters were re-evaluated from the archive slides. According to the Lauren classification, there were intestinal, diffuse and mixed types. Tumour stage was determined based on the American Joint Commission for Cancer criteria (AJCC 8th edition).

Formalin-fixed and paraffin wax-embedded gastric adenocarcinoma blocks were obtained from the Department of Pathology archive. They were stained with haematoxylin-eosin. For the immunohistochemical staining process, 3- $\mu$ m-thick sections were taken from the formalin-fixed paraffin-embedded tissues paraffin blocks. CD44 and CD133 were stained in a Leica Bond Max staining device in compliance with immunohistochemical staining datasheets. The immunohistochemically stained slides were examined by two pathologists. The CD44 (MRQ-13) monoclonal antibody (1: 200 dilution; clone MRQ-13, Millipore Sigma, USA) immunohistochemical stain was applied. Inflammatory cells were used for the positive internal control of CD44. ${ }^{5}$ The CD133 (D4W4N) XP Rabbit mAb (1: 500 dilution; clone D4W4N, Cell Signalling Technology) immunohistochemical stain was applied. Colon adenocarcinoma was used for CD133 control in accordance withits datasheet. Haematoxylin-eosinand immunohistochemical sections were examined under a Leica DM 2000 LED light microscope. They were photographed with an INFINITY 3ANALYZE Release 6.5 imaging system.

Immunohistochemical staining was semi-quantitatively evaluated in membranous staining for CD44 and cytoplasmic, membranous or luminal staining for CD 133. According to staining intensity, negative staining was scored as 0 , weak staining was scored as $1+$ (minimal, in less than $10 \%$ of tumour cells), medium level positive staining was scored as $2+$, and intense staining was scored as $3+{ }^{5,10,11}$ According to the staining extent, less than $<5 \%$ staining was scored as $0,>5-25 \%$ staining was scored as $1,>25-50 \%$ staining was scored as $2,>50-75 \%$ staining was scored as 3 , and $>75 \%$ staining was scored as $4 .^{11}$ The total score (IHK) was obtained as staining intensity $x$ staining extent.

The quantitative data are summarised using medians $\left(25^{\text {th }}-75^{\text {th }}\right.$ percentile), while frequencies (percentages) are given for the qualitative data. Mann-Whitney U-test and Kruskal-Wallis test were used for group comparisons. For pairwise comparisons, post-hoc Dunn's tests were used. The results were assessed on the significance level of $p<0.05$. The analyses were performed using the Statistical Package for the Social Sciences version 22.0 for Windows (SPSS Inc., Chicago, Illinois, USA). This study was authorised in advance by the Ethics Committee of Bolu Abant Izzet Baysal University for clinical studies (Decision No: 2018/123).

\section{RESULTS}

All clinicopathological parameters, as well as CD44 and CD133 immunohistochemical staining, are summarised in Tablel.

No CD44 staining was observed in $2.4 \%(n=2)$ of the 83 cases. The CD44 staining intensity was one positive $(+)$ in $13.3 \%(n=$ $11)$ of the cases, two positive $(++)$ in $18.1 \%(n=15)$ of the cases and three positive $(+++)$ in $66.3 \%(n=55)$ of the cases. The CD44 staining extent was one positive $(+)$ in $21.7 \%(n=18)$ of the cases, two positive $(++)$ in $22.9 \%(n=19)$, three positive $(+++)$ in $21.7 \%(n=18)$ of the cases and four positive $(++++)$ in $31.3 \%$ $(n=26)$ of the cases.

No CD44 staining was observed in $1.2 \%(n=1)$ of the 83 cases. The CD133 staining intensity was one positive (+) in $13.3 \%$ $(n=11)$ of the cases, two positive $(++)$ in $20.5 \%(n=17)$ of the cases and three positive $(+++)$ in $64.1 \%(n=54)$ of the cases. The CD44 staining extent was one positive $(+)$ in $20.5 \%(n=17)$ of the cases, two positive $(++)$ in $19.3 \%(n=16)$, three positive $(+++)$ in $32.5 \%(n=27)$ of the cases and four positive $(++++)$ in $26.5 \%$ $(n=22)$ of the cases.

The CD44 and CD133 immunohistochemical expressions in the intestinal and diffuse type adenocarcinomas are shown in Figures 1 and 2. Considering the CD44 and CD133 expressions in pathological $\mathrm{T}$, the study was found significantly more intense expression in the T3 and T4 cases $(p=0.009, p=0.002)$. The pairwise comparisons showed significantly higher CD44 and CD133 expressions in T3 and T4a in comparison to T1b.

The CD44 expressions were found to be significantly different between the pathological $\mathrm{N}$ groups $(p=0.008)$. Cases without lymph node metastasis had expressions, but on very low levels. No significant difference was found between the pathological $\mathrm{N}$ groups in terms of CD133 expression ( $p=0.158)$. Additionally, no significant difference was found in the pathological metastasis (M) groups in terms of CD44 and CD133.

According to pathological staging, the CD44 and CD133 expressions were the lowest in stage 1 and differed in other stages. However, no significant differences were found between these expression rates $(p=0.075, p=0.084)$.

No significant difference was found in the CD44 and CD133 expressions in terms of gender, age, type of surgical operation, tumour location and size. No significant correlation was found between CD44 and CD133 expressions and intestinal metaplasia, helicobacter pyloripresence, and tumour periphery lymphocyte ratio. 
Table I: All clinicopathological parameters with CD44 and CD133 immunohistochemical staining.

\begin{tabular}{|c|c|c|c|c|c|c|}
\hline & $\mathbf{n}$ & $\%$ & $\begin{array}{c}\text { CD44 } \\
\text { (intensity and extent) }\end{array}$ & $\mathbf{p}$ & $\begin{array}{c}\text { CD133 (intensity and } \\
\text { extent) }\end{array}$ & $\mathbf{p}$ \\
\hline Gender & & & & 0.539 & & 0.335 \\
\hline Female & 25 & 30.1 & $6(2.5-12)$ & & $6(2.5-10.5)$ & \\
\hline Male & 58 & 69.9 & $7.5(3.8-12)$ & & $9(6-12)$ & \\
\hline Age & & & & 0.829 & & 0.256 \\
\hline$\leq 60$ & 24 & 28.9 & $9(2.3-12)$ & & $9(6-12)$ & \\
\hline$>60$ & 59 & 71.1 & $6(4-12)$ & & $6(3-12)$ & \\
\hline Lauren type & & & & 0.001 & & 0.032 \\
\hline Intestinal & 57 & 68.7 & $6(2-9)^{a}$ & & $6(2-9)$ & \\
\hline Diffuse & 20 & 24.1 & $12(9-12)^{a}$ & & $9(6-12)$ & \\
\hline Mix & 6 & 7.2 & $7.5(5.3-12)$ & & $10.5(6-12)$ & \\
\hline Histological grade & & & & 0.043 & & 0.014 \\
\hline 1 & 12 & 14.5 & $3(1-9)^{a}$ & & $2(1-6)^{a, b}$ & \\
\hline 2 & 30 & 36.1 & $6(4-12)$ & & $6(4-12)^{a}$ & \\
\hline 3 & 41 & 49.4 & $9(4-12)^{a}$ & & $9(6-12)^{b}$ & \\
\hline Pathological M & & & & 0.464 & & 0.199 \\
\hline MO & 53 & 63.9 & $6(3.5-12)$ & & $6(3-9)$ & \\
\hline M1 & 30 & 36.1 & $9(2.8-12)$ & & $9(6-12)$ & \\
\hline Pathological N & & & & 0.008 & & 0.258 \\
\hline 0 & 23 & 27.7 & $4(2-6)^{a}$ & & $6(2-9)$ & \\
\hline 1 & 17 & 20.5 & $6(3.5-10.5)$ & & $6(3-12)$ & \\
\hline 2 & 10 & 12.0 & $9(3.8-12)$ & & $7.5(5.5-12)$ & \\
\hline $3 a$ & 19 & 22.9 & $12(6-12)^{a}$ & & $9(6-12)$ & \\
\hline $3 b$ & 14 & 16.9 & $9(5.3-12)$ & & $9(5.5-9.8)$ & \\
\hline Pathological T & & & & 0.009 & & 0.002 \\
\hline la & 3 & 3.6 & 1 (NA) & & 2 (NA & \\
\hline $1 b$ & 13 & 15.7 & $4(1-6)^{a, b}$ & & $2(1.5-6)^{a, b}$ & \\
\hline 2 & 2 & 2.4 & 9 (NA) & & 9 (NA) & \\
\hline 3 & 25 & 30.1 & $9(6-12)^{a}$ & & $9(6-10.5)^{a}$ & \\
\hline $4 a$ & 34 & 41.0 & $9(4-12)^{b}$ & & $9(5.5-12)^{b}$ & \\
\hline $4 b$ & 6 & 7.2 & $10.5(1.8-12)$ & & $9(2.5-12)$ & \\
\hline Stage & & & & 0.075 & & 0.084 \\
\hline $1 a$ & 12 & 14.5 & $3(1-6)$ & & $3(1.3-6)$ & \\
\hline $1 b$ & 2 & 2.4 & 3.5 (NA) & & 5.5 (NA) & \\
\hline $2 a$ & 5 & 6 & $9(5-12)$ & & $9(3-12)$ & \\
\hline $2 b$ & 12 & 14.5 & $7.5(4-11.3)$ & & $9(6-9)$ & \\
\hline $3 a$ & 8 & 9.6 & $7.5(3.3-12)$ & & $6(1.8-12)$ & \\
\hline $3 b$ & 7 & 8.4 & $12(6-12)$ & & $9(1-12)$ & \\
\hline $3 c$ & 7 & 8.4 & $9(6-12)$ & & $9(6-12)$ & \\
\hline 4 & 30 & 36.1 & $9(2.8-12)$ & & $9(6-12)$ & \\
\hline
\end{tabular}




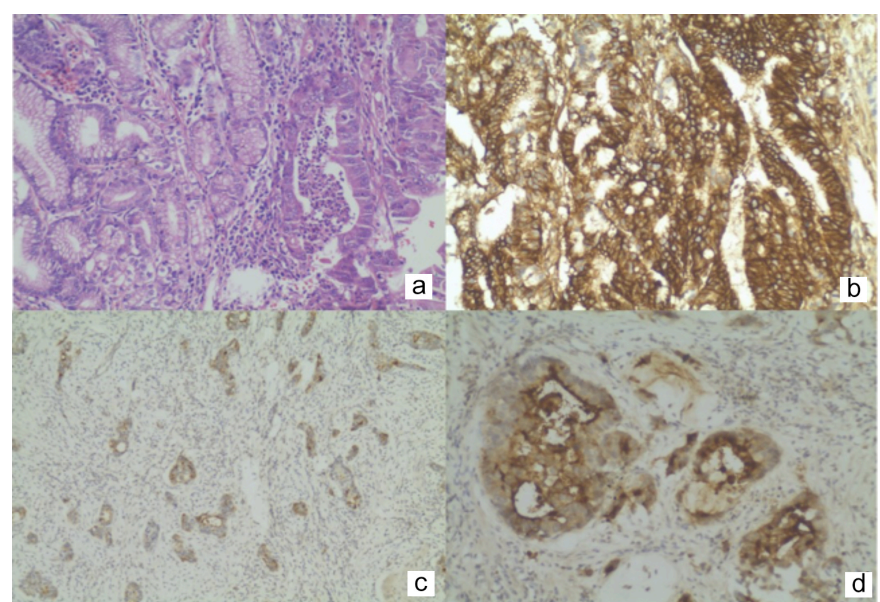

Figure 1: (a) Adenocarcinoma, intestinal type HEX200, (b) Diffuse membranous expression of CD44, X200, (c) Cytoplasmic expression of CD133, X100, (d) Luminal expression of CD133, X200.

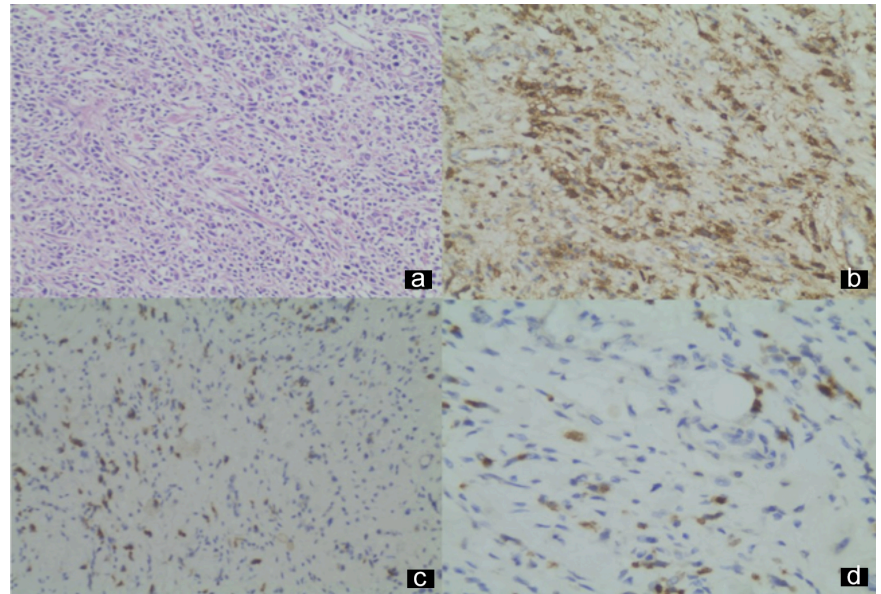

Figure 2: (a) Adenocarcinoma, diffuse type, HEX200, (b) Diffuse membranous expression of CD44, X200, (c) Cytoplasmic expression of CD133, X200, (d) Cytoplasmic expression of CD133, X400.

\section{DISCUSSION}

Gastric adenocarcinomas are divided into intestinal and diffuse subtypes with different epidemiological and prognostic factors, according to the Lauren histological classification, and $90 \%$ of the gastric cancers are adenocarcinomas. $^{3,12}$

CD44 expression is associated with lymph node metastasis, poor prognosis and distant metastasis in gastric cancer. ${ }^{13,14}$ In-vivo and in-vitro studies have shown that CD44+ stem cells have self-renewal and differentiation ability, and they have emphasised that these cells may be used as a gastric cancer stem cell marker. ${ }^{3}$

Mayer et al. found that CD44 expression in primer gastric tumour occurred in 30 out of 61 cases $(49 \%) .{ }^{15}$ CD44 expression in primary tumours was found to be correlated with tumour differentiation, tumour metastasis, recurrence and tumour-related mortality. ${ }^{15}$

Jung et al. found that CD44 expression was an independent prognostic factor, and poor prognosis was associated with old age, high pathological stage and poorly differentiated histology.

Ghaffarzadehgan et al. determined that CD44 expression was related to stage and affected survival separately from stage. ${ }^{12}$ It may contribute to the diagnosis of gastric cancer and may be a marker in determining tumour metastasis. ${ }^{12}$ Ibrahim et al. observed that CD44-positive expression was related to tumour differentiation grade, tumour recurrence, short disease-free survival and overall survival. ${ }^{16}$ Nosrati et al. found that CD44 was associated with intestinal type cancer, tumours with sizes of 4-8 cm, moderate differentiation and serous invasion without lymphovascular invasion. ${ }^{2}$

Wu et al. conducted the first systematic review on the prognostic role of the CD44 family in gastric cancer. ${ }^{7}$ While the expression of CD44s was found to be related to the $\mathrm{N}$ stage and distant metastasis, no correlation was found between the expression of CD44s and T stage or TNM stage. ${ }^{7}$

In this study, while CD44 was associated with histological grade, intestinal type, lymphovascular and perineural invasion, pathological $\mathrm{T}$ and pathological $\mathrm{N}$, there was no correlation with pathological M. According to these results, it was considered that CD44 could be a predictive factor that may be used in prediction of pathological $\mathrm{T}$ and $\mathrm{N}$ in biopsy materials.

Ishigami et al. reported that CD133 expression was related to $T$ stage, nodal involvement, venous invasion and advanced stage $(p<0.01) .{ }^{9}$ In addition to the presence of CD133, detection of CD133-positive tumour cell volume was emphasised to have prognostic significance. ${ }^{9}$ Similarly, we assessed the extent and intensity of the immunohistochemical stains separately.

Zhao et al. examined 336 gastric adenocarcinoma cases, where CD133 expression was related to tumour size, invasion depth and lymph node metastasis. ${ }^{11}$ Yu et al. found that CD133 was related to $>5 \mathrm{~cm}$ tumour size, lymph node metastasis, advanced TNM stage, and lymphovascular invasion. ${ }^{17}$

In this study, while CD133 was associated with histological grade, intestinal type and pathological $T$, there were no correlations with pathological N, pathological M, lymphovascular invasion or perineural invasion. CD133 may be used as a prognostic factor in prediction of tumour invasion depth, thanks to its relationship with pathological T stage.

Meta-analysis studies emphasised that CD133 alone has no prognostic significance, and it should be evaluated in combination with other cancer stem cell markers such as CD44. ${ }^{18}$ Wakamatsu et al. examined 190 gastric cancer cases and determined that CD44 and CD133 were associ- 
ated with TNM stage, and they were independent prognostic factors. ${ }^{6}$ CD44-positive gastric cancer cases were found to be associated with advanced $N$ stage $(p=0.04)$ and TNM stage $(p<0.001) .{ }^{6}$ CD133 gastric cancer cases were found to be associated with $\mathrm{N}$ stage $(p=0.02)$ and intestinal tumour subtype. ${ }^{6}$

This study observed a low expression levels at low TNM stages, but it did not find a significant correlation. While a correlation similar to this study existed between CD44 and $\mathrm{N}$ stage, no significant correlation was found with CD133.

The lowest level of staining was detected in stage 1 in both immunohistochemical staining procedures, but no significant correlation was found with stage, which was similar to the study by Wu et al. ${ }^{7}$ It was important that both markers had low expression in stage 1 . None of these markers was correlated with metastasis, and there is no evidence that shows a correlation with metastasis in the literature.

\section{CONCLUSION}

Although CD44 is regarded to be associated with more prognostic parameters in comparison to CD133, both immunohistochemical stains were shown to be related to pathological tumour stage. Thus, these may be guiding for determining the tumour depth. Moreover, it is predicted that they may be used as prognostic factors on targeted gene treatments to increase the effectiveness of treatment in gastric adenocarcinoma.

\section{FUNDING INFORMATION:}

This study was supported by Bolu Abant Izzet Baysal University Scientific Research Projects (2018.08.19.1385).

\section{ETHICAL APPROVAL:}

Approval was obtained from Ethics Committee of Bolu Abant Izzet Baysal University for Clinical studies. (Decision No. 2018/123).

\section{PATIENTS' CONSENT:}

As this study was retrospective, the patients' consents were waived.

\section{CONFLICT OF INTEREST:}

The authors declared no conflict of interest.

\section{AUTHORS' CONTRIBUTION:}

All authors have done conception or design of the work or the acquisition analysis, or interpretation of data for the work, drafting the work or revising critically for important intellectual content, final approval of the version to be published.

\section{REFERENCES}

1. Dhingra S, Feng W, Brown RE, Zhou Z, Khoury T, Zhang R, et al. Clinicopathologic significance of putative stem cell markers, CD44 and nestin, in gastric adenocarcinoma. Int J Clin Exp Pathol 2011; 4(8):733-41.

2. Nosrati A, Naghshvar F, Khanari S. Cancer stem cell markers CD44, CD133 in primary gastric adenocarcinoma. Int J Mol Cell Med 2014; 3(4):279-86.

3. Singh SR. Gastric cancer stem cells: A novel therapeutic target. Cancer Lett 2013; 338(1):110-9. doi.org/10.1016/ j.canlet.2013.03.035.

4. Turkey Cancer Statistics. Turkish public heal inst 2017.

5. Wang T, Ong CW, Shi J, Srivastava S, Yan B, Cheng CL, et al. Sequential expression of putative stem cell markers in gastric carcinogenesis. Br J Cancer 2011; 105(5):658-65. dx.doi.org/10.1038/bjc.2011.287.

6. Wakamatsu Y, Sakamoto N, Oo HZ, Naito Y, Uraoka N, Anami $\mathrm{K}$, et al. Expression of cancer stem cell markers ALDH1, CD44 and CD133 in primary tumor and lymph node metastasis of gastric cancer. Pathol Int 2012; 62(2):112-9. doi: 10.1111/j.1440-1827.2011.02760.x.

7. Wu Y, Li Z, Zhang C, Yu K, Teng Z, Zheng G, et al. CD44 family proteins in gastric cancer: A meta-analysis and narrative review. Int J Clin Exp Med 2015; 8(3):3595-606.

8. Jung WY, Kang Y, Lee H, Mok YJ, Kim HK, Kim A, et al. Expression of moesin and CD44 is associated with poor prognosis in gastric adenocarcinoma. Histopathol 2013; 63(4):474-81. doi: 10.1111/his.12202.

9. Ishigami S, Ueno S, Arigami T, Uchikado Y, Setoyama T, Arima $\mathrm{H}$, et al. Prognostic impact of CD133 expression in gastric carcinoma. Anticancer Res 2010; 30(6):2453-7.

10. Lee $\mathrm{HH}$, Seo KJ, An CH, Kim JS, Jeon HM. CD133 expression is correlated with chemoresistance and early recurrence of gastric cancer. J Surg Oncol 2012; 106(8):999-1004. doi: 10.1002/jso.23178.

11. Zhao P, Li Y, Lu Y. Aberrant expression of CD133 protein correlates with $\mathrm{Ki}-67$ expression and is a prognostic marker in gastric adenocarcinoma. BMC Cancer 2010; 10:218. doi: 10.1186/1471-2407-10-218.

12. Ghaffarzadehgan K, Jafarzadeh M, Raziee $H R$, Sima $H R$, Esmaili-Shandiz E, Hosseinnezhad $H$, et al. Expression of cell adhesion molecule CD44 in gastric adenocarcinoma and its prognostic importance. World J Gastroenterol 2008; 14(41):6376-81. doi: 10.3748/wjg.14.6376.

13. Xia $\mathrm{P}, \mathrm{Xu} \mathrm{XY}$. Prognostic significance of CD44 in human colon cancer and gastric cancer: Evidence from bioinformatic analyses. Oncotarget 2016; 7(29). doi: 10.18632/oncotarget.9998.

14. Liu YJ, Yan PS, Li J, Jia JF. Expression and significance of CD44s, CD44v6, and nm23 mRNA in human cancer. World J Gastroenterol 2005; 11(42):6601-6. doi: 10.3748/wjg. v11.i42.6601.

15. Mayer B, Jauch KW, Günthert U, Figdor CG, Schildberg FW, Funke I, et al. De-novo expression of CD44 and survival in gastric cancer. Lancet 1993; 342(8878):1019-22. doi: 10.1016/0140-6736(93)92879-x.

16. Ibrahim HM, AbdElbary AM, Mohamed SY, Elwan A, Abdelhamid MI, Ibrahim A. Prognostic value of cyclin D1 and CD44 expression in gastric adenocarcinoma. J Gastrointest Cancer 2019; 50(3):370-9. doi: 10.1007/ s12029-018- 
0079-2.

17. Yu J, Zhang P, Wu J, Wu S, Li X, Wang S, et al. Expressions and clinical significances of CD133 protein and CD133 mRNA in primary lesion of gastric adenocacinoma. J Exp Clin Cancer Res 2010; 29(1):141. doi: 10.1186/1756-9966-
29-141.

18. Saricanbaz I, Karahacioglu E, Ekinci O, Bora H, Kilic D, Akmansu M. Prognostic significance of expression of CD133 and ki-67 in gastric cancer. Asian Pacific J Cancer Prev 2014; 15(19):8215-9. doi: 10.7314/apjcp.2014.15.19.8215. 